\title{
Differential expression of cell cycle and WNT pathway-related genes accounts for differences in the growth and differentiation potential of Wharton's jelly and bone marrow-derived mesenchymal stem cells
}

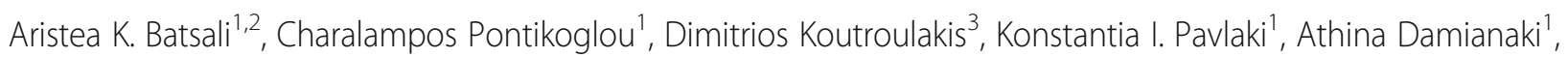
Irene Mavroudi ${ }^{1}$, Kalliopi Alpantaki ${ }^{4}$, Elisavet Kouvidi ${ }^{1}$, George Kontakis ${ }^{4}$ and Helen A. Papadaki ${ }^{*}$

\begin{abstract}
Background: In view of the current interest in exploring the clinical use of mesenchymal stem cells (MSCs) from different sources, we performed a side-by-side comparison of the biological properties of MSCs isolated from the Wharton's jelly (WJ), the most abundant MSC source in umbilical cord, with bone marrow (BM)-MSCs, the most extensively studied MSC population.

Methods: MSCs were isolated and expanded from BM aspirates of hematologically healthy donors $(n=18)$ and from the WJ of full-term neonates $(n=18)$. We evaluated, in parallel experiments, the MSC immunophenotypic, survival and senescence characteristics as well as their proliferative potential and cell cycle distribution. We also assessed the expression of genes associated with the WNT- and cell cycle-signaling pathway and we performed karyotypic analysis through passages to evaluate the MSC genomic stability. The hematopoiesis-supporting capacity of MSCs from both sources was investigated by evaluating the clonogenic cells in the non-adherent fraction of MSC co-cultures with BM or umbilical cord blood-derived $\mathrm{CD}_{3} 4^{+}$cells and by measuring the hematopoietic cytokines levels in MSC culture supernatants. Finally, we evaluated the ability of MSCs to differentiate into adipocytes and osteocytes and the effect of the WNT-associated molecules WISP-1 and SFRP4 on the differentiation potential of WJ-MSCs.

(Continued on next page)
\end{abstract}

\footnotetext{
* Correspondence: e.papadaki@uoc.gr

'University of Crete School of Medicine, Heraklion, Greece

Full list of author information is available at the end of the article
} 
(Continued from previous page)

Results: Both ex vivo-expanded MSC populations showed similar morphologic, immunophenotypic, survival and senescence characteristics and acquired genomic alterations at low frequency during passages. WJ-MSCs exhibited higher proliferative potential, possibly due to upregulation of genes that stimulate cell proliferation along with downregulation of genes related to cell cycle inhibition. WJ-MSCs displayed inferior lineage priming and differentiation capacity toward osteocytes and adipocytes, compared to BM-MSCs. This finding was associated with differential expression of molecules related to WNT signaling, including WISP1 and SFRP4, the respective role of which in the differentiation potential of WJ-MSCs was specifically investigated. Interestingly, treatment of WJ-MSCs with recombinant human WISP1 or sFRP4 resulted in induction of osteogenesis and adipogenesis, respectively. WJ-MSCS exhibited inferior hematopoiesis-supporting potential probably due to reduced production of stromal cell-Derived Factor-1 a, compared to BM-MSCs.

Conclusions: Overall, these data are anticipated to contribute to the better characterization of WJ-MSCs and BM-MSCs for potential clinical applications.

Keywords: Bone marrow, Wharton's jelly, Mesenchymal stem cells, Cell cycle, WNT pathway

\section{Background}

In the past 16 years, the field of mesenchymal stem (stromal) cells (MSCs) has progressed at a great pace, as their exceptional characteristics are being unraveled and encouraging data from preclinical and clinical studies accumulate. In this regard, MSC-based treatment is now considered as a promising modality in the therapeutic intervention of various diseases or tissue damage [1].

Bone marrow (BM)-derived MSCs have been considered as the gold standard for clinical applications. However, there is currently great interest in identifying alternative, more easily accessible sources of MSCs. In this context, umbilical cord (UC), a medical waste obtained with a painless, simple and safe procedure during delivery, has drawn much attention as an attractive substitute of the BM for MSC isolation [2].

MSC origin may have an important impact in the biological properties of the cells and may even affect their effectiveness in cell-based therapies [3]. As regards to differences between BM- and UC-derived MSCs, contradictory results have been published so far, probably due to discrepancies in the isolation protocols of UC-MSCs in different studies [4]. Interestingly, MSCs from different UC compartments have been reported to possess different biological properties [5].

In view of the current interest in exploring the use of UC-MSCs from different sources in the clinic, the proper characterization of the cells is of particular importance. In the present study, we have performed a side-by-side comparison of MSCs isolated from the Wharton's jelly (WJ), the most abundant source of MSCs in UC [4], with BM-MSCs, the most extensively studied adult MSC population [3]. Specifically, we have comparatively studied the biological properties of the WJ-MSCs and BM-MSCs, namely their proliferative potential, the survival and senescence characteristics, the differentiation potential toward osteocytes and adipocytes and their capacity to support hematopoiesis. We have also explored the cell cycle distribution of the two MSC populations, in association with the expression of cell cycle-related genes. Because the WNT pathway has been involved in the proliferation and differentiation process of MSCs from different sources, we have probed the expression of genes related to the WNT signaling and the effect of WNT-related molecules in the differentiation capacity of WJ-MSCs. The results of the study have pointed out significant differences in the biological properties of BM- and WJ-derived MSCs that should be taken into consideration when choosing the best MSC source for a given application.

\section{Methods \\ Samples}

UC samples were obtained from 18 full-term neonates (38 to 39 weeks) following normal labor. BM samples were obtained from 18 hematologically healthy individuals (nine males and nine females, median age 50 years) undergoing orthopedic surgery for hip replacement. The study has been approved by the Ethics Committee of the University Hospital of Heraklion and informed consent for the UC and the BM donation was obtained from the neonate mothers and the individuals undergoing surgery, respectively, according to the Helsinki Protocol.

\section{MSC cultures}

MSCs were isolated from BM and WJ as previously described and expanded in appropriately supplemented alpha-Minimum Essential Medium ( $\alpha$-MEM; Gibco Invitrogen, Paisley, Scotland), at $37{ }^{\circ} \mathrm{C} / 5 \% \mathrm{CO}_{2}$ fully humidified atmosphere [6-8]. The culture medium was replaced twice per week and on $80-90 \%$ confluency, MSCs were detached using $0.25 \%$ Trypsin-1mM EDTA (Gibco, Invitrogen), reseeded at a concentration of $2000 \mathrm{cells} / \mathrm{cm}^{2}$ and further expanded for a total of ten passages $(\mathrm{P})[6,7]$. 
Cell-free supernatants from WJ- and BM-MSC cultures at P2, P6 and P10 were stored at $-80{ }^{\circ} \mathrm{C}$ for quantification of FMS-like tyrosine kinase 3 ligand (FLT3L; Quantikine, R\&D Systems, Minneapolis, MN, USA), Granulocyte- Colony Stimulating Factor (high sensitivity human G-CSF; Quantikine, R\&D Systems), Stromal Cell-derived Factor- $1 \alpha$ (human CXCL12/SDF-1 $\alpha$; Quantikine, R\&D Systems) and Granulocyte Macrophage-Colony Stimulating Factor (human GM-CSF; Affymetrix, eBioscience, San Diego, CA, USA), by means of enzyme-linked immunosorbent assay.

\section{Immunophenotype and survival characteristics of MSCs}

Trypsinized MSCs from P2 to P10 were immunophenotypically characterized by flow cytometry using anti-human monoclonal antibodies (mAb) against CD29 (4B4; CytoStat/Beckman Coulter, Fullerton, CA, USA), CD44 (J173; Immunotech/Beckman Coulter, Marseille, France), CD73 (AD2; BD Biosciences Pharmingen, San Diego, CA, USA), CD90 (F15.42; Immunotech/ Beckman Coulter), CD105 (SN6; Caltag, Burlingame, CA, USA), anti-CD146 (P1H12; BD Biosciences Pharmingen), CD45 (IMMU19.2; Immunotech/ Beckman Coulter), CD14 (RMO52; Immunotech/ Beckman Coulter), CD34 (QBend10; Beckman Coulter), CD31 (5.6E; Immunotech/Beckman Coulter), CD19 (J3119; Immunotech/Beckman Coulter) and HLA-DR (Immu-357, Immunotech/Beckman Coulter), as previously described [6].

Flow cytometry was also used to study the apoptotic characteristics of MSCs at two representative passages (P2 and P6) by means of 7-aminoactinomycin D staining (7-AAD; Calbiochem-Novabiochem, Nottingham, UK) as previously described [9]. Results were expressed as proportions of 7-AAD ${ }^{\text {neg }}$ (live), 7-AAD ${ }^{\text {dim }}$ (early apoptotic) and $7-\mathrm{AAD}^{\text {bright }}$ (late apoptotic) cells. Acquisition and analysis were performed in a Cytomics FC 500 flow cytometer (Beckman Coulter, Brea, CA, USA) on a minimum of 10,000 events.

\section{Cell cycle analysis of MSCs}

For cell cycle analysis, BM- and WJ-MSCs from P2, at a confluency of $80-90 \%$ were trypsinized, washed with PBS and fixed with $70 \%$ cold ethanol. Cells were treated with $0.5 \mathrm{mg} / \mathrm{mL}$ RNase A (PureLink RNase A; Invitrogen) and stained with $50 \mu \mathrm{g} / \mathrm{mL}$ propidium iodide (PI, Invitrogen) in the dark for $30 \mathrm{~min}$ at $37^{\circ} \mathrm{C}$. DNA content was determined by flow cytometry and the percentage of cells in different phases of cell cycle was assessed. A minimum of 100,000 events were acquired per sample.

\section{Proliferative potential of MSCs}

The proliferative potential of MSCs was evaluated by a 3-[4,5-dimethylthiazol-2-yl]-2,5 diphenyl tetrazolium bromide (MTT)-based assay at P2 and by estimating the population doubling (PD) time through P2-P10
$[6,7,10]$. The formula $2^{n}=\mathrm{Nx} /$ No was used to calculate the PDs of cells at each passage based on the number of cells counted in the flask after trypsinization $(\mathrm{Nx})$ and the number of cells initially plated (No) $[6,7,10]$. Cumulative PDs were also calculated as previously described [11].

\section{Assessment of MSC senescence \\ Evaluation of senescence-associated gene expression}

A real-time reverse transcription polymerase chain reaction (RT-PCR) was used for the evaluation of the expression of genes associated with cell senescence, namely the senescence-associated cyclin-dependent kinase (CDKN) inhibitors $C D K N 1 A(p 21), C D K N 2 A(p 16)$ and $C D K N 2 B$ ( $p 15)$, the tumor suppressors TP53 and retinoblastoma $(R B 1)$, and the phosphate-associated RhoGAP proteintyrosine (PARG1) in P2, P6, and P10 MSCs, as previously described $[6,7,10]$. Glyceraldehyde-3-phosphate dehydrogenase $(G A P D H)$ was used as internal control gene and gene expression values were expressed as $2^{-\Delta \mathrm{Ct}}$, where $\Delta \mathrm{Ct}=\mathrm{Ct}$ gene of interest $-\mathrm{Ct}{ }^{\mathrm{GAPDH}}$.

\section{MSC relative telomere length measurement}

DNA was extracted from P2, P6, and P10 MSCs using the QIAamp DNA mini kit (Qiagen, Hilden, Germany) and the relative telomere length was evaluated using a previously described semi-quantitative real-time PCR method with $\beta$-globin as control single-copy gene [12]. The relative telomere length was expressed as $2^{-\Delta \mathrm{Ct}}$ $\left(\Delta \mathrm{Ct}=\mathrm{Ct}{ }^{\text {telomere }}-\mathrm{Ct}^{\beta \text {-globin }}\right)$.

\section{Senescence-associated $\beta$-galactosidase (SA- $\beta$-gal) expression}

Expression of SA- $\beta$-gal at P2, P6 and P10 MSCs was determined by a chromogenic assay as previously described $[7,13]$. The number of SA- $\beta$-gal-positive MSCs was evaluated under a phase-contrast microscope per 100 consecutively counted MSCs. All experiments were performed in triplicate.

\section{MSC differentiation assays}

Trypsinized WJ- and BM-MSCs from P2 were induced to differentiate into adipocytes and osteocytes as previously described $[6,10,14]$. Adipogenesis was assessed by Oil Red $\mathrm{O}$ and osteogenesis by Alizarin Red and Von Kossa staining $[6,14]$. Furthermore, total RNA from BM- and WJ-MSCs following differentiation at P2 toward adipocytes and osteocytes was isolated, reverse transcribed and subsequently amplified by real-time RT-PCR, for the quantification of genes related to adipogenesis, namely peroxisome proliferator activated receptor- $\gamma(P P A R G)$ and CCAAT/enhancerbinding protein alpha $(C E B P A)$, and osteogenesis, namely alkaline phosphate $(A L P)$, osteocalcin (OSC), distal-less homeobox protein $5(D L X 5)$ and runt-related transcription 
factor 2 (RUNX2). GAPDH was used as internal control gene. The primer sequences and real-time RT-PCR detailed conditions have been described previously [12].

In a set of experiments, adipogenesis- or osteogenesisrelated gene expression in WJ-MSCs was evaluated by real-time RT-PCR following differentiation of P2 cells in the presence or absence of $20 \mathrm{nM}$ recombinant human (rh)-secreted frizzled related protein 4 (rh-sFRP4, R\&D Systems) or $50 \mathrm{ng} / \mathrm{ml} \mathrm{rh-WNT1-inducible-signaling} \mathrm{path-}$ way protein 1 (rh-WISP1, R\&D Systems), respectively.

\section{Cytogenetic analysis of MSCs}

Conventional cytogenetic analysis of BM- and WJMSCs was performed at P2, P6 and P8 as previously described [15-17]. MSC metaphases were identified using trypsin-Giemsa (GTG) banding and 15 to 25 metaphase cells were analyzed and classified according to the International System for Human Cytogenetic Nomenclature [16]. A chromosomal aberration was defined as clonal abnormality when at least two metaphases were demonstrating the same structural rearrangement or chromosome gain, whereas a chromosome loss had to be identified in at least three metaphases $[15,16]$.

\section{WNT signaling pathway and cell cycle PCR arrays}

Total RNA was isolated from BM-MSC $(n=6)$ and WJMSC $(n=6)$ cultures at P2 as previously described [12]. By using the human WNT Signaling Pathway $\mathrm{RT}^{2}$ Profiler $^{\text {ra }}$ PCR Array (SABiosciences, Qiagen) we profiled the expression of 84 genes related to WNT-mediated signal transduction. Similarly, using the human Cell Cycle $\mathrm{RT}^{2}$ Profiler $^{\mathrm{mu}}$ PCR Array (SABiosciences, Qiagen) we profiled the expression of 84 genes related to cell cycle signaling pathway. The fold change (FC) for each gene between the group of WJ-MSCs and the group of BM-MSCs was calculated with the $\Delta \Delta \mathrm{Ct}$ method $\left(\mathrm{FC}=2^{-\Delta \Delta \mathrm{Ct}}\right)$. At least a two-fold difference in gene expression between WJ- and BM-MSCs was considered significant [12].

\section{Validation of the WNT PCR array results by real-time RT-PCR}

To validate the PCR - array results, significantly down- and over-expressed genes were further assessed in BM- and WJ-MSCs (P2) by real-time RT-PCR (SYBR GreenER qPCR Supermix; Invitrogen), using the specific primers of the PCR array for WISP1, sFRP4 and GAPDH (SABiosiences, Qiagen). Reactions were performed in Rotor-Gene 6000 using a two-step cycling program consisting of 45 cycles of $95{ }^{\circ} \mathrm{C}$ for 3 seconds and $60{ }^{\circ} \mathrm{C}$ for 30 seconds. A melting curve $\left(62-95^{\circ} \mathrm{C}\right)$ was generated at the end of each run to verify specificity of the reactions.

\section{Evaluation of the hematopoiesis-supporting capacity of MSCs}

A previously described two-stage culture procedure was used to test the capacity of WJ- and BM-MSCs to support normal hematopoiesis [12]. In brief, confluent MSC stromal layers from WJ and BM samples, grown in $25 \mathrm{~cm}^{2}$ flasks, were irradiated (10 Gy), recharged with immunomagnetically sorted (Miltenyi Biotec, Bergisch Gladbach, Germany) normal allogeneic BM- or UC blood (UCB)-derived $\mathrm{CD} 34^{+}$cells $\left(5 \times 10^{4}\right)$ and kept in $10 \mathrm{~mL}$ appropriately supplemented Iscove's modified Dulbecco's medium (Invitrogen) at $37{ }^{\circ} \mathrm{C} / 5 \% \mathrm{CO}_{2}$ fully humidified atmosphere. At weekly intervals for a total of 3 weeks, cultures were fed by demi-depopulation and the non-adherent cells (NACs) were counted and assayed for clonogenic progenitor cells, namely granulocyte colony-forming units (CFU-G), macrophage CFU (CFU-M), granulocyte-macrophage CFU (CFUGM), and erythroid - CFU (CFU-E), as previously described $[12,18,19]$. The colonies were finally defined as total myeloid, that is, total CFU-GM (CFU-G plus CFU-M plus CFU-GM), CFU-E, and total CFU (total CFU-GM plus CFU-E) [12, 18, 19].

\section{Statistical analysis}

Data were analyzed using the GraphPad Prism Statistical PC program (GraphPad Software, San Diego, CA, USA). Grouped data were expressed as mean \pm 1 standard deviation and compared by means of the non-parametric Mann-Whitney $U$ test. The two-way analysis of variance was used to define differences between WJ-MSCs and BM-MSCs in PD time, gene expression and cytokine levels through passages as well as in CFU numbers in culture supernatants time course and in optical density obtained by MTT at P2. The chi-square test was used for the evaluation of differences between WJ-MSCs and BM-MSCs in the frequency of cytogenetic aberrations through passages.

\section{Results}

BM- and WJ-MSCs exhibit similar morphologic and immunophenotypic characteristics

BM- and WJ-derived MSCs were successfully expanded and serially reseeded for ten passages. Cultured MSCs from both sources displayed the characteristic spindlelike morphology and the immunophenotypic analysis throughout P2-P10 demonstrated that cultures constituted of a homogenous cell population positive for CD73, CD90, CD146, CD105, CD29, CD44 and negative for CD31, CD19, CD45, CD14, CD34 and HLA-DR surface antigens. No difference was identified between BM- and WJ-MSCs in the expression of any of the above markers. 
BM-MSCs grow at a slower rate compared to WJ-MSCs The PD time in BM-MSC cultures was significantly increased in comparison to WJ-MSCs throughout P2P10. Specifically, as shown in Fig. 1a, the PD time ranged from $3.88 \pm 1$ days (P2) to $10.9 \pm 1.7$ days (P10) in BM-MSCs versus $1.88 \pm 1.18$ days (P2) to $4 \pm$ 1.6 days $(\mathrm{P} 10)$ in WJ-MSCs $(P<0.0001)$. In accordance with these findings are the results obtained by the MTT assay at a representative passage (P2), which showed that the number of metabolically active BMMSCs, corresponding to the obtained optical density, remained significantly lower during the 21-day culture period, compared to WJ-MSCs $(P<0.0001)$ (Fig. 1b). Finally, the individual long-term growth curve data depicted in Fig. 1c, clearly show the inferior proliferative potential of BM-MSCs compared to WJ-MSCs. Taken together, these findings suggest that BM-MSCs grow at a significant slower rate compared to WJMSCs.
BM- and WJ-MSCs display similar survival characteristics through passages

To investigate whether the difference in the growth rate between BM- and WJ-MSCs is associated with altered survival characteristics, we evaluated the proportion of apoptotic cells at P2 and P6 by means of 7-AAD expression. No statistically significant differences were identified, however, in the proportion of apoptotic cells (expressed as the sum of 7-AAD ${ }^{\text {dim }}$ and 7-AAD ${ }^{\text {bright }}$ cells) between BMand WJ-MSCs at either P2 $(8.42 \pm 2.6 \%$ and $7.9 \pm 1.92 \%$, respectively) or P6 (7.7 $\pm 4.66 \%$ and $6 \pm 3.25 \%$, respectively).

Since replicative cell senescence has been associated with defective proliferative capacity, we investigated whether culture-expanded BM-MSCs are more prone to cellular senescence compared to WJ-MSCs. To this end, we evaluated SA- $\beta$-gal activity, telomere length and senescence-associated gene expression through passages, as indices of cell senescence. The number of SA- $\beta$-galpositive cells at P2, P6 and P10 was significantly higher

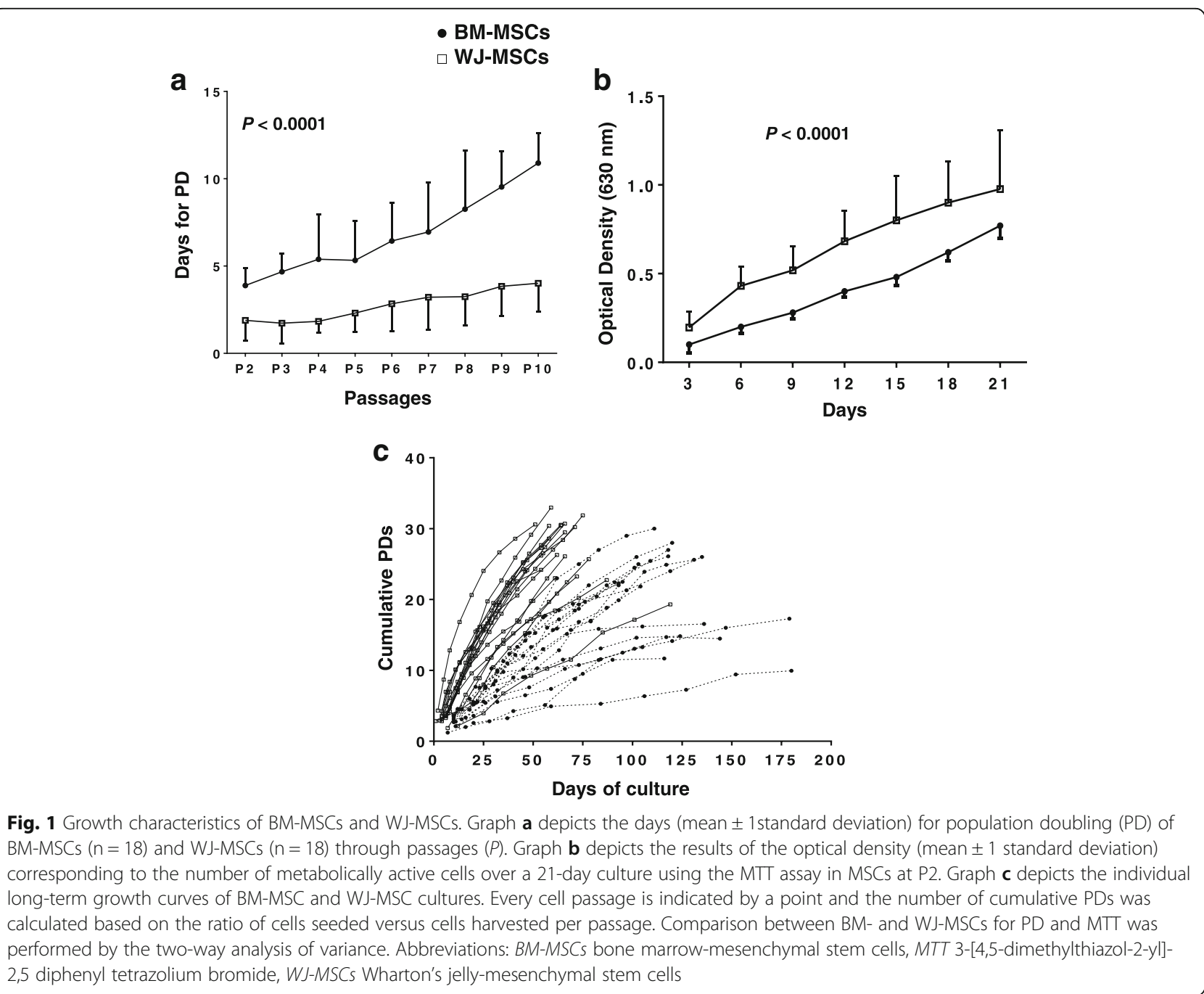


in BM- compared to WJ-MSCs $(P=0.0169, P=0.0327$ and $P=0.0001$, respectively) (Table 1 ). Telomere length was consistently shortened in both BM- and WJ-MSC cultures during passages and the mean relative telomere length of both BM- and WJ-MSCs at P2 was significantly higher compared to P10 $(P=0.0208$ and $P=$ 0.0002 , respectively) (Table 1 ). However, no statistically significant difference was detected between BM- and WJ-MSC cultures in the mean telomere length of cells at P2, P6 and P10 suggesting that the reduced proliferative potential of BM-MSCs compared to WJ-MSCs cannot be attributed to accelerated telomere loss. As regards to senescence-associated gene expression, no significant difference was identified between BM- and WJMSCs in PARG1, RB1, CDKN1A and CDKN2A gene expression whereas a statistically significant decrease was obtained in CDKN2B and TP53 gene expression in BMMSCs throughout P2-P6-P10 $(P<0.0001$ and $P<0.0001$, respectively) (Fig. 2). Taking together all the above data, and taking into consideration that SA- $\beta$-gal activity is neither causative nor prerequisite for manifestation of senescence [20,21], we conclude that the inferior proliferative potential of BM-MSCs compared to WJ-MSCs cannot be attributed to premature senescence.

\section{Upregulation of cell cycle-related genes in WJ-MSCs}

We next investigated whether the above described differences in the proliferative characteristics of BM- and WJMSCs might be associated with differences in cell cycle distribution of cells in the culture. Indeed, at the representative P2, the proportion of cells in the quiescent G1 phase was significantly higher in BM-MSC cultures compared to WJ-MSCs $(93.25 \pm 1.63 \%$ and $89.54 \pm 1.45 \%$, respectively; $P$ $<0.0001$ ) (Fig. 3a). In contrast, the percentage of cells in $S$ and $\mathrm{G} 2 / \mathrm{M}$ phase in BM-MSC cultures $(2.9 \pm 2.4 \%$ and 3.85 $\pm 1.66 \%$, respectively) was significantly lower compared to WJ-MSCs $(3.34 \pm 1.27 \%$ and $7.11 \pm 0.98 \%$, respectively) ( $P$ $=0.0212$ and $P<0.0001$, respectively) (Fig. 3a). To probe further the differences in the cell cycle distribution of cells in BM- and WJ-MSC cultures, we subsequently investigated the expression of genes related to cell cycle, by means of a PCR array. By considering as significant an at least two-fold difference in gene expression between WJ- and BM-MSCs [6-8], we found that 13 out of 84 cell cyclerelated genes were differentially expressed between the two MSC populations at P2 (Fig. 3b). More specifically, a significant increase in aurora kinase $\mathrm{B}$ gene $(A U R K B$; FC $=3.1)$ expression was observed in WJ-MSCs. This gene encodes for a protein that is associated with microtubules during chromosome movement and segregation. Cyclin D2 (CCND2) and cell division cycle 25 homologue A $(C D C 25 A)$ gene expression was also upregulated in WJMSCs compared to BM-MSCs $(\mathrm{FC}=4.5$ and $\mathrm{FC}=2.5$, respectively). Both genes encode for proteins that regulate cell cycle G1/S transition. Furthermore, several genes encoding for cell cycle regulators of $\mathrm{G} 2 / \mathrm{M}$ transition were also increased in WJ-MSCs, namely CCNA2 $(\mathrm{FC}=2.3), C C N B 1$ $(\mathrm{FC}=2.0)$, a gene encoding for a protein that binds to the catalytic subunit of cyclin-dependent kinases $(\mathrm{CDC} 28$ protein kinase regulatory subunit $2, C K S 2 ; \mathrm{FC}=2.6$ ), a cyclindependent kinase activator (cell division cycle 25C, $C D C 25 C$; $F C=4.2)$ and a gene involved in microtubuleassociated process $(C D C 20 ; \mathrm{FC}=5)$. Moreover, MKI67 gene, which encodes for a nuclear protein which is associated with cellular proliferation, was also increased in WJMSCs $(F C=2.8)$. In contrast, a significant decrease was obtained in WJ-MSCs compared to BM-MSCs in the expression of the antiapoptotic gene BCL2 $(\mathrm{FC}=-4)$ which mediates delayed transition into $\mathrm{S}$ phase as well as in the cyclin-dependent kinase inhibitor-1B $(C D K N 1 B ; \mathrm{FC}=-2.2)$, which mediates $\mathrm{G} 1$ arrest. An upregulation of $C D K N 2 B$ $(\mathrm{FC}=3.08)$ that mediates $\mathrm{G} 1$ arrest and upregulation of ataxia-telangiectasia mutated gene $(A T M ; \mathrm{FC}=4.03)$ that mediates a cell cycle delay after DNA damage, was observed in WJ-MSCs compared to BM-MSCs. Taken together all the above data suggest that WJ-MSCs display mostly an upregulation of genes that stimulate cell proliferation along with a concomitant downregulation of genes related to cell cycle inhibition, compared to BM-MSCs.

\section{Reduced adipogenic and osteogenic differentiation potential of WJ-MSCs}

To compare the adipogenic and osteogenic potential of WJ- and BM-MSCs, we induced in parallel adipogenesis

Table 1 Senescence characteristics and telomere length of WJ-MSCs and BM-MSCS

\begin{tabular}{|c|c|c|c|c|c|c|}
\hline \multirow[t]{2}{*}{ Passages } & \multicolumn{3}{|c|}{ SA- $\beta$-gal positive cells $(\%)^{a}$} & \multicolumn{3}{|c|}{ Mean telomere length ${ }^{a}$} \\
\hline & WJ-MSCS & BM-MSCS & $P$ & WJ-MSCS & BM-MSCS & $P$ \\
\hline$\overline{P 2}$ & $10.28 \pm 5.73$ & $25.82 \pm 17$ & 0.0169 & $11,958 \pm 1687$ & $10,530 \pm 2244$ & N.S. \\
\hline P6 & $15.14 \pm 9.73$ & $39.33 \pm 22.46$ & 0.0327 & $10,128 \pm 1317$ & $8901 \pm 870.9$ & N.S. \\
\hline P10 & $24.09 \pm 11$ & $59.14 \pm 15.69$ & 0.0001 & $8529 \pm 1619$ & $8212 \pm 1486$ & N.S. \\
\hline
\end{tabular}

Comparisons in each indicated passage ( $P$; P2, P6, P10) between WJ-MSCs $(n=18)$ and BM-MSCs $(n=18)$ have been performed by the nonparametric Mann-Whitney $U$ test and the statistically significant $P$ values are shown

Abbreviations: WJ-MSCs Wharton's jelly-mesenchymal stem cells, BM-MSCs bone marrow-mesenchymal stem cells, SA- $\beta$-gal senescence-associated $\beta$ galactosidase, N.S. no statistically significant difference

${ }^{\mathrm{a}}$ The data are expressed as means \pm 1 standard deviation 

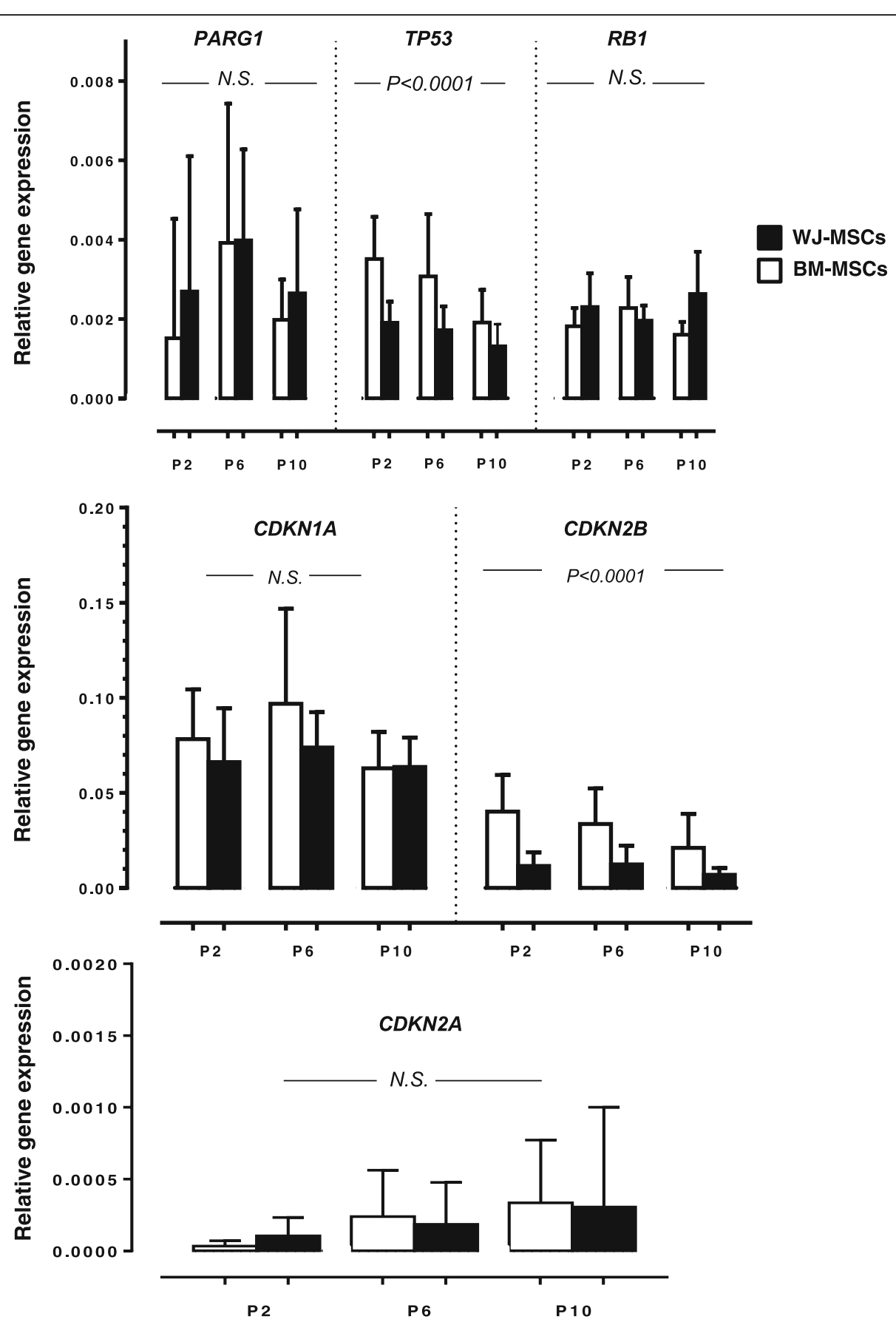

Fig. 2 Senescence-associated gene expression in BM-MSCs and WJ-MSCs. The bars represent the relative expression (mean +1 standard deviation) of the senescence-associated genes PARG1, TP53, RB1, CDKN1A, CDKN2A, and CDKN2B in BM-MSCs $(n=18)$ and WJ-MSCs $(n=18)$ through passages (P). Gene expression analysis was performed by means of real-time RT-PCR using GAPDH as internal control gene ( $2^{-\triangle \mathrm{Ct}}$ method). Statistical analysis has been performed by the two-way analysis of variance to compare multiple mean values through P2-P6-P10. Abbreviations: BM-MSCs bone marrow-mesenchymal stem cells, N.S. no statistically significant difference, WJ-MSCs Wharton's jelly-mesenchymal stem cells

and osteogenesis in P2 MSCs from both sources and assessed the specific staining reaction and the expression of adipocyte- and osteocyte-specific genes. WJ-MSCs displayed reduced adipogenic differentiation potential compared to BM-MSCs as indicated by the Oil Red O staining and by the decreased mRNA expression of PPARG and CEBPA ( $P=0.0002$ and $P=0.0005$, respectively) (Fig. 4a). WJ-MSCs did not qualitatively differ from BM-MSCs in their capacity to differentiate into osteocytes, as indicated by Alizarin Red and Von Kossa 

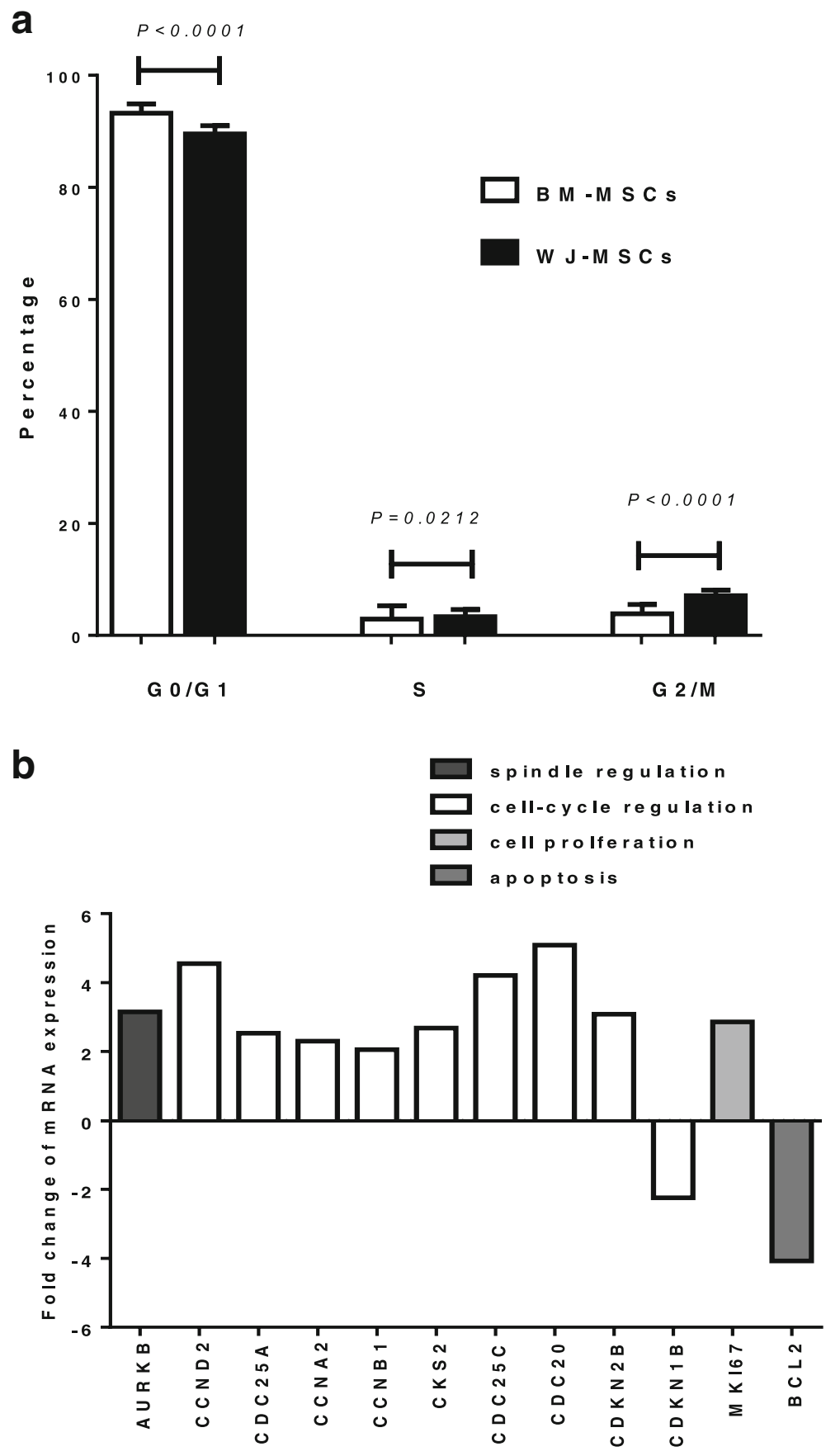

Fig. 3 Cell cycle analysis and relative expression of cell cycle-related genes in WJ-MSCs compared to BM-MSCs. a BM- and WJ-MSCs from P2, at a confluency of $80-90 \%$ were stained by propodium iodide and subsequently cell cycle distribution was analyzed by flow cytometry. Bars represent the proportion of cells in each phase of the cell cycle and the values shown are the means \pm 1 standard deviation of $n=18$ independent experiments. b The columns represent the mean fold change in the expression of genes related to cell cycle in WJ-MSCs $(n=6)$ at P2, compared to the respective BM-MSCs $(n=6)$, using a real-time PCR array. The fold change for each gene between the group of WJ-MSCs and the group of BM-MSCS was calculated with the $\Delta \Delta \mathrm{Ct}$ method and only genes exhibiting at least a two-fold change are depicted. Differentially expressed genes have been grouped according to their implication in specific cellular process such as spindle regulation, cell cycle regulation, cell proliferation, apoptosis and DNA damage response. Abbreviations: BM-MSCs bone marrow-mesenchymal stem cells, P passage, WJ-MSCS Wharton's jelly-mesenchymal stem cells

staining (Fig. 4b). However, the mRNA levels of RUNX2, $D L X 5, A L P$ and OSC were significantly reduced in WJMSCs during osteogenic differentiation as compared to
BM-MSCs $(P<0.0001, P<0.0001, P<0.0001$ and $P<$ 0.0097 , respectively) (Fig. 4b). Interestingly, undifferentiated WJ-MSCs (P2) expressed significantly lower levels 


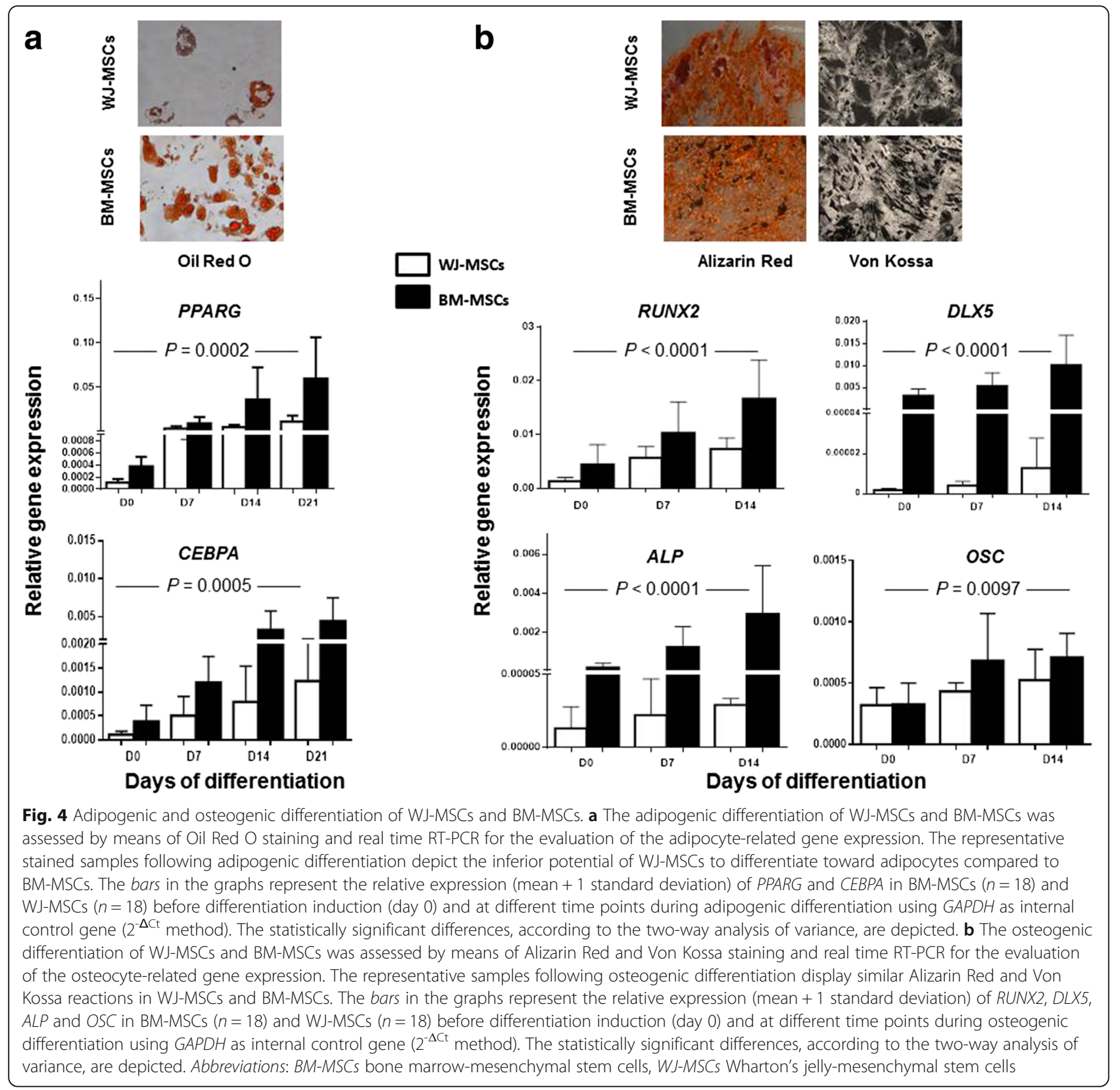

of PPARG, CEBPA, RUNX2, DLX5 and ALP compared to BM-MSCs $(P=0.0002, P=0.0281, P=0.0141, P<$ 0.0001 and $P<0.0001$, respectively), suggesting that the WJ-MSCs are less primed toward the adipocytic and osteoblastic lineages compared to BM-MSCs.

\section{Differential expression of WNT signaling molecules between WJ- and BM-MSCs}

The WNT pathway is involved in the differentiation and proliferation process of MSCs [22]. We have therefore evaluated the expression of genes encoding proteins of the WNT pathway in WJ- and BM-MSCs using a PCR array. We found that expression of 25 out of 84 WNT- related genes displayed at least a two-fold difference between WJ- and BM-MSCs (Fig. 5a). Specifically, five genes encoding for WNT proteins were significantly downregulated in WJ-MSCs (WNT3A, FC =-2.4; WNT5A, FC $=-2.3 ; \quad W N T 5 B, \quad \mathrm{FC}=-3.4 ; \quad W N T 7 B, \quad \mathrm{FC}$ $=-2.6$; WNT8A, FC = -2.2) as well as four genes encoding for WNT receptors (FZD1, FC $=-4 ; \mathrm{FZD} 3, \mathrm{FC}$ $=-11.4 ; F Z D 4, \mathrm{FC}=-5.4 ; F Z D 5, \mathrm{FC}=-3.2)$. Two genes implicated in the canonical WNT signaling were downregulated $(A X I N 2, \mathrm{FC}=-2.6$; porcupine homologue 1 PORCN1, FC =-2.4), whereas two genes involved in the non-canonical WNT signaling were upregulated (disheveled-associated activator of morphogenesis 1 -DAAM1, 


\section{a}
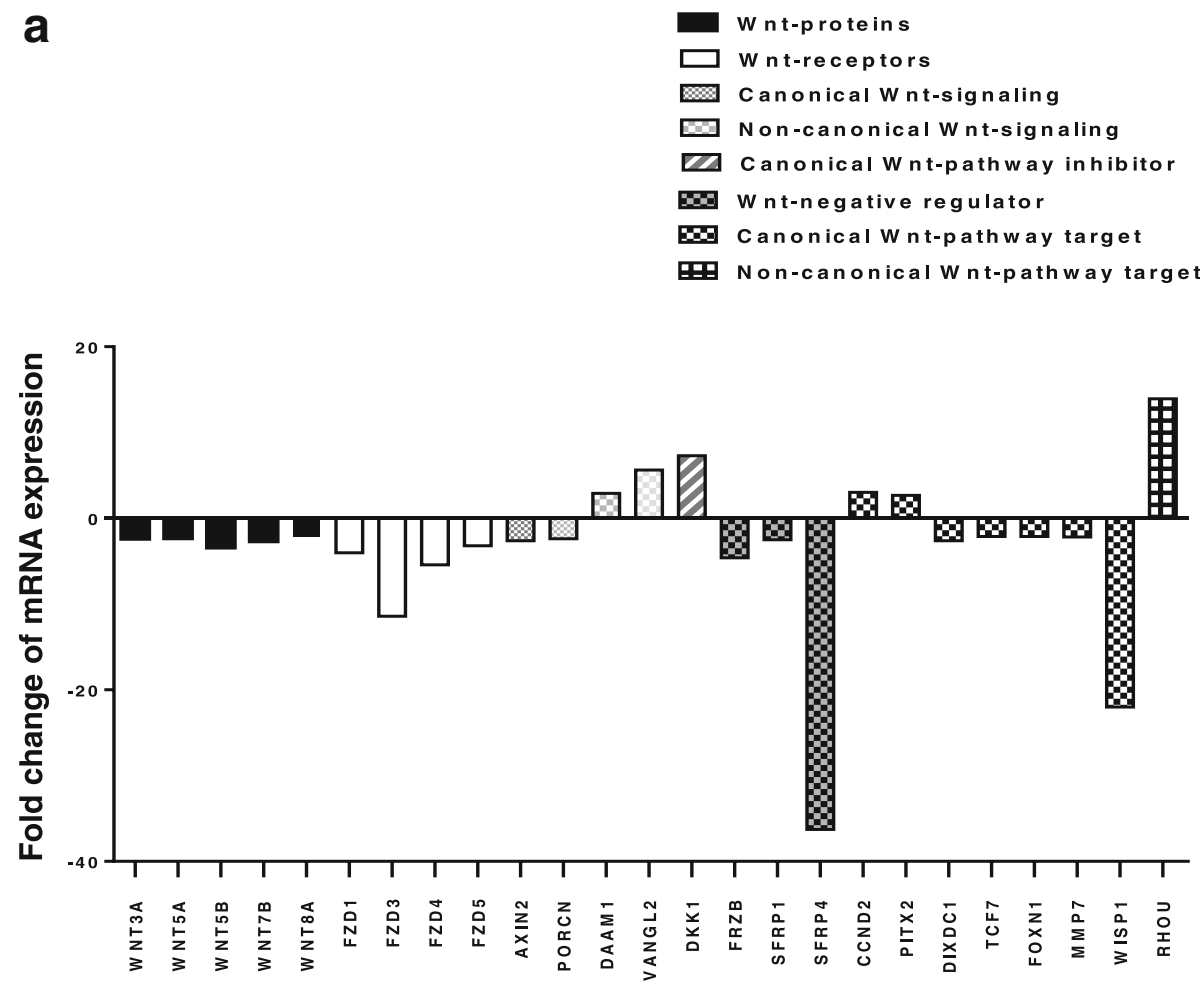

b

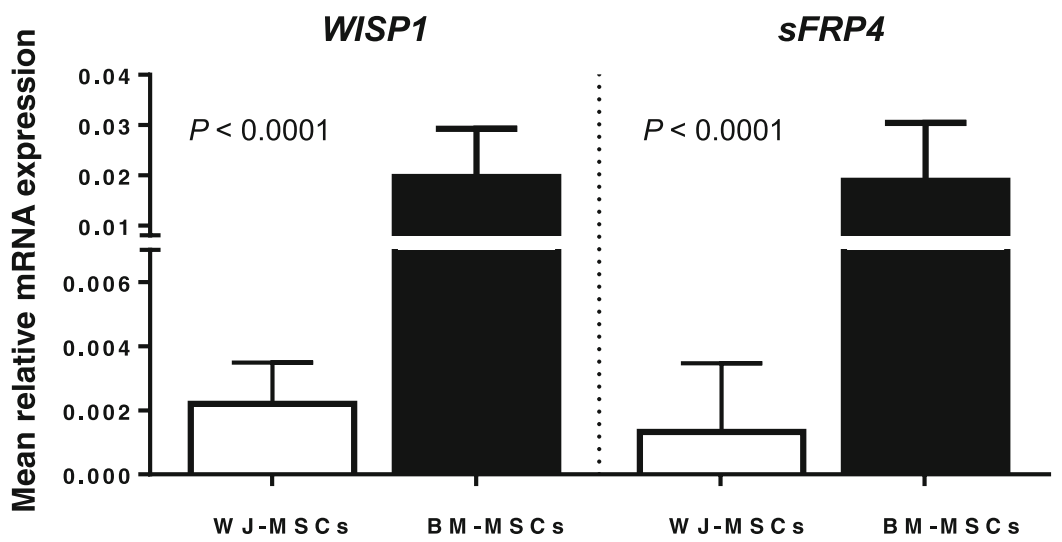

Fig. 5 Relative expression of genes related to WNT pathway in WJ-MSCs and BM MSCs. a The columns represent the mean fold change of genes involved in WNT signaling in P2 WJ-MSCs $(n=6)$ as compared to BM-MSCs $(n=6)$ using a real-time PCR array. Only genes exhibiting at least a two-fold change in their expression are depicted. $\mathbf{b}$ Validation of the array. The bars represent the relative mRNA expression (mean +1 standard deviation) of WISP1 and SFRP4 in WJ-MSCs $(n=18)$ and BM-MSCs $(n=18)$ at P2 using real-time RT-PCR. Values correspond to $2^{-\triangle C t}$ using GAPDH as control gene. Comparisons between WJ- and BM-MSCs were performed by the nonparametric Mann-Whitney $U$ test and the $P$ values are depicted. Abbreviations: BM-MSCS bone marrow-mesenchymal stem cells, sFRP4 secreted frizzled related protein 4, WISPI WNT1-inducible-signaling pathway protein 1, WJ-MSCS Wharton's jelly-mesenchymal stem cells

$\mathrm{FC}=2.9 ;$ Vang-like 2 -VANGL2, FC = 5.6) in WJ-MSCs. The gene encoding for the canonical WNT pathway inhibitor dickkopf-1 (DKK1) was significantly upregulated $(\mathrm{FC}=7.3)$, while the WNT negative regulators $F R Z B$, sFRP1 and sFRP4 were decreased $(F C=-4.6, F C=-2.5$, $\mathrm{FC}=-36.2$, respectively) in WJ-MSCs. The canonical WNT pathway targets DIX domain containing 1 (DIXDC1), transcriptional factor-7 (TCF7), forkhead box protein N1 (FOXN1), matrix metalloproteinase-7 (MMP7) and WISP1 were significantly downregulated in $\mathrm{WJ}-\mathrm{MSCs}(\mathrm{FC}=-2.6, \mathrm{FC}=-2.1, \mathrm{FC}=-2.1, \mathrm{FC}=-2.2$ and $\mathrm{FC}=-22$, respectively). The expression of CCND2 and its activator Pituitary homeobox 2 (PITX2), which also encode targets of the canonical WNT pathway were significantly increased in WJ-MSCs $(\mathrm{FC}=3$; and $\mathrm{FC}=2.7$, respectively). Finally, the expression of the non- 
canonical WNT target Ras Homolog Family Member U (RHOU) gene was significantly increased in WJ-MSCs $(\mathrm{FC}=13.9)$.

Taken together, all the above data suggest that the canonical WNT pathway is downregulated in WJ-MSCs as evidenced by the downmodulation of canonical WNTs, WNT receptors and canonical WNT targets, as well as the upregulation of the $\mathrm{WNT} / \beta$-catenin signaling inhibitor $D K K 1$. We speculate that the reduced expression of the WNT inhibitors/negative regulators $S F R P 1, F R Z B$ (sFRP3), sFRP4 and AXIN2 may represent a compensatory phenomenon aiming to upregulate the canonical WNT signaling. The upregulation of genes involved in the non-canonical WNT pathway in WJ-MSCs is also anticipated because the canonical and non-canonical WNT signaling are generally antagonistic [23].

To validate the results obtained from the PCR array, we evaluated the expression of genes with the greatest FC, namely WISP1 and $s F R P 4$, by real-time RT-PCR in P2 WJ-MSCs $(\mathrm{n}=18)$ and BM-MSCs $(\mathrm{n}=18)$. In accordance with the PCR array, the mean relative mRNA expression of both WISP1 and sFRP4 was significantly decreased in WJ-MSCs $(0.0022 \pm 0.0013$ and $0.0013 \pm$ 0.002 , respectively) compared to BM-MSCs $(0.019 \pm$ 0.009 and $0.018 \pm 0.01$, respectively) $(P<0.0001$ and $P<$ 0.0001, respectively) (Fig. 5b).

\section{sFRP4 is implicated in the adipogenic differentiation of WJ-MSCs}

Secreted-FRP4 is a WNT inhibitor that directly binds WNTs, antagonizing therefore both canonical and noncanonical WNT pathways [24]. It has been reported that rh-sFRP4 may induce adipogenesis of adipose tissuederived MSCs in vitro [25]. We have therefore investigated whether the profound downregulation of SFRP4 mRNA expression in WJ-MSCs compared to BM-MSCS (FC $=-36.2$ ), might explain the decreased adipocytic differentiation potential of the cells, at least in part. We first evaluated the sFRP4 gene expression during adipogenic differentiation of WJ-MSCs $(n=5)$ and we indeed found a gradual statistically significant increase in mRNA levels' time course (Fig. 6a). Specifically, sFPR4 mRNA expression at day 14 was significantly higher compared to baseline and day $7(P=0.022$ and $P=0.022$, respectively).

We next cultured WJ-MSCs in adipocytic medium for 7 days in the absence or presence of $20 \mathrm{nM}$ rh-sFRP4 and evaluated the mRNA levels of the adipogenesisspecific genes PPARG and CEBPA. As shown in Fig. 6b, at day 7 of adipogenic differentiation, WJ-MSC cultures treated with rh-sFRP4 displayed significant upregulation of the above genes compared to untreated cultures $(P=$ 0.012 and $P=0.003$, respectively) or cultures prior to adipogenic induction $(P=0.0002$ and $P<0.0001$, respectively).

\section{WISP1 is implicated in the osteogenic differentiation of WJ-MSCs}

It has been shown that WISP1 promotes the osteogenic differentiation of BM-MSCs and WISP1 gene expression has been reported to increase during BM-MSC osteogenic differentiation in vitro [26-28]. In accordance with these reports, we also observed a statistically significant upregulation of WISP1 mRNA expression in WJ-MSCs during osteogenic differentiation (Fig. 6a). Specifically, WISP1 mRNA expression at day 14 was significantly higher compared to baseline and day $7(P=0.0095$ and $P=0.0286$, respectively).

To investigate whether the downregulation of WISP1 mRNA expression in WJ-MSCs compared to BM-MSCs might have a role in the reduced osteogenic differentiation potential of WJ-MSCs, we cultured WJ-MSCs $(n=5)$ in osteogenic medium for 7 days in the absence or presence of $50 \mathrm{ng} / \mathrm{ml} \mathrm{rh-WISP1}$ and we next evaluated the mRNA levels of the osteogenesis-specific genes RUNX2, ALP and OSC. As shown in Fig. 6c, rh-WISP1 treatment resulted in a significant upregulation of these genes compared to untreated WJ-MSCs $(P=0.026, P=0.0232$ and $P=0.0079$, respectively) or compared to WJ-MSCs prior to osteogenic induction $(P=0.0002, P=0.0004$ and $P=0.0043$, respectively).

\section{WJ-MSCs exhibit inferior hematopoiesis-supporting potential than BM-MSCs}

To evaluate the capacity of WJ- and BM-MSCs to support the hematopoietic progenitor cell growth, we assessed the clonogenic potential of the NAC fraction of irradiated (P2) confluent WJ-MSC $(n=6)$ and BM-MSC $(n=6)$ cultures. The frequency of the total CFUs obtained by the NACs was significantly lower in cultures with feeder layers from WJ-MSCs compared to BMMSCs, irrespectively of the source of the recharging $\mathrm{CD}^{+} 4^{+}$cells, i.e., BM $(P=0.0008)$ (Fig. $\left.7 \mathrm{a}\right)$ or $\mathrm{UCB}(P<$ 0.0001) (Fig. 7b). More specifically, the increased CFU numbers in cultures of BM-MSCs compared to the respective of WJ-MSCs, was due to the increased number of both CFU-E and total CFU-GM progenitor cells derived from either BM-CD34 ${ }^{+}$cells $(P=0.0435$ and $P=$ 0.0001 , respectively) (Fig. 7a) or $\mathrm{UCB}-\mathrm{CD} 34^{+}$cells $(P=$ 0.0007 and $\mathrm{P}<0.0001$, respectively) (Fig. 7b). Interestingly, both WJ- and BM-MSC feeder layers exhibited superior hematopoiesis-supporting capacity when recharged with $\mathrm{UCB}-\mathrm{CD} 34^{+}$cells than $\mathrm{BM}-\mathrm{CD} 34^{+}$cells, as indicated by the evaluation of the total CFU numbers $(P<0.0001$ and $P<0.0001$, respectively).

To probe further the hematopoiesis-supporting potential of WJ- and BM-MSCs, we analyzed several common hematopoietic cytokines, namely FLT3L, SDF-1 $\alpha$, G-SCF and GM-CSF in P2, P6 and P10 supernatants from WJMSC $(n=18)$ and BM-MSC $(n=18)$ cultures. As shown in Table 2, FLT3L levels did not differ significantly 


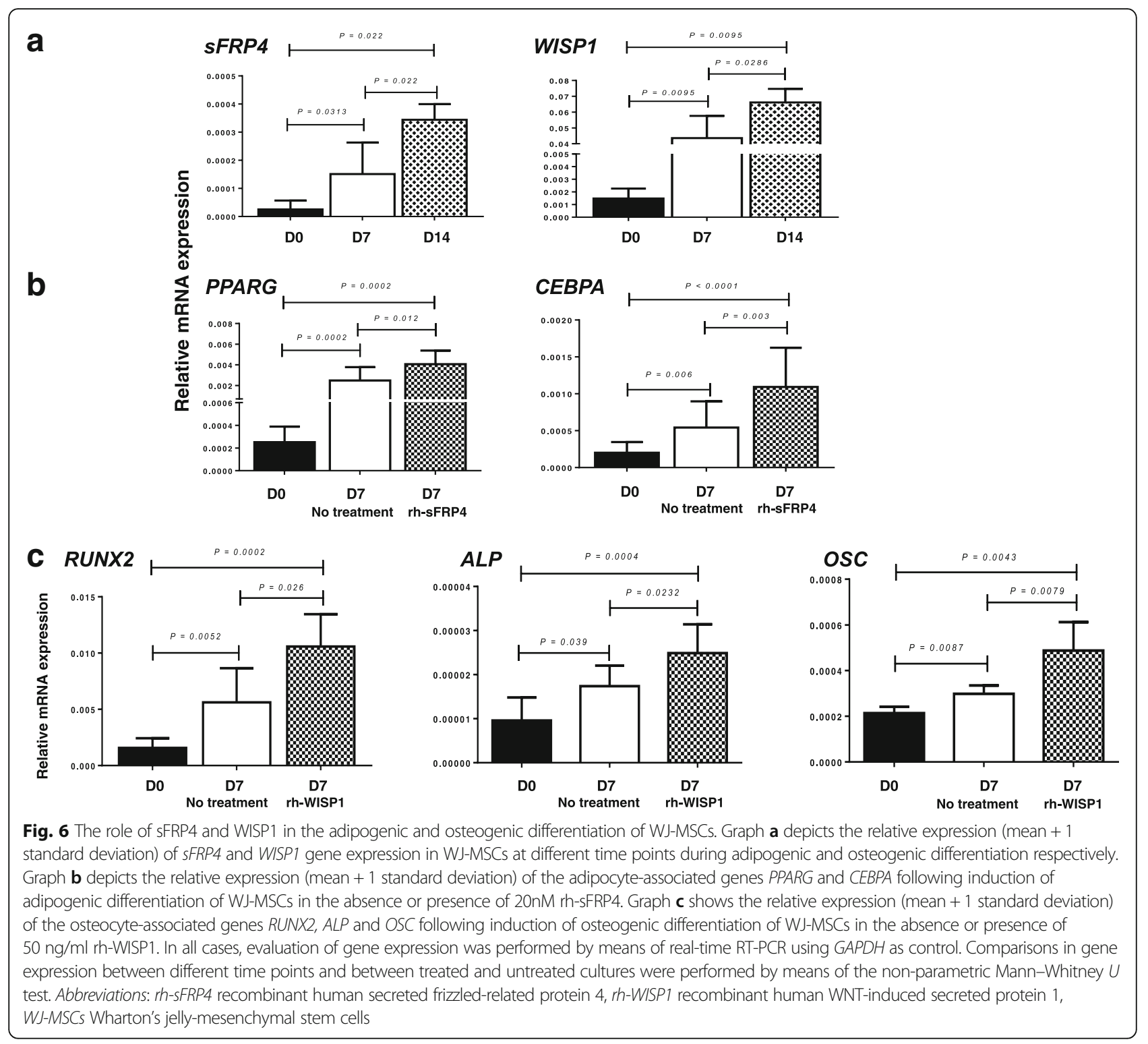

between WJ- and BM-MSC cultures whereas SDF- $1 \alpha$ levels were significantly decreased in WJ-compared to BM-MSC culture supernatants throughout passages $(P$ $=0.0012)$. G-CSF and GM-CSF were detectable in WJMSC supernatants through passages but were undetectable in the respective BM-MSC cultures $(P<0.0001$ and $P<0.0001$, respectively). These data suggest that the inferior hematopoiesis-supporting capacity of WJ-MSCs compared to BM-MSCs is unlikely to be associated with impaired production of hematopoiesis-inducing cytokines, but may be associated with the lower production of SDF- $1 \alpha$, at least in part.

\section{Discussion}

WJ-derived MSCs have gained much attention over the last years for potential use in cell-based therapies, since they can be easily isolated without any ethical concerns from a tissue which is discarded after birth [3]. In the present study, we have performed a rigorous side-by-side comparison of WJ-MSCs with BM-MSCs, which represents the most extensively studied MSC population. We even excluded the possible contamination of WJ-MSCs by diverse MSC populations from different UC regions, i.e., perivascular and sub-amnion, by stripping the UC vessels before cutting the WJ into pieces and use them as explants for culture establishment. Various biological properties of the two MSC populations were comparatively studied in parallel experiments, including the expression of cell cycle and WNT pathway-related genes, which have never been studied before. The involvement of WNT pathway-related molecules in the differentiation capacity of WJ-MSCs were also studied for the first time. 


\section{a Recharges with $\mathrm{BM}$-derived $\mathrm{CD} 34^{+}$cells}
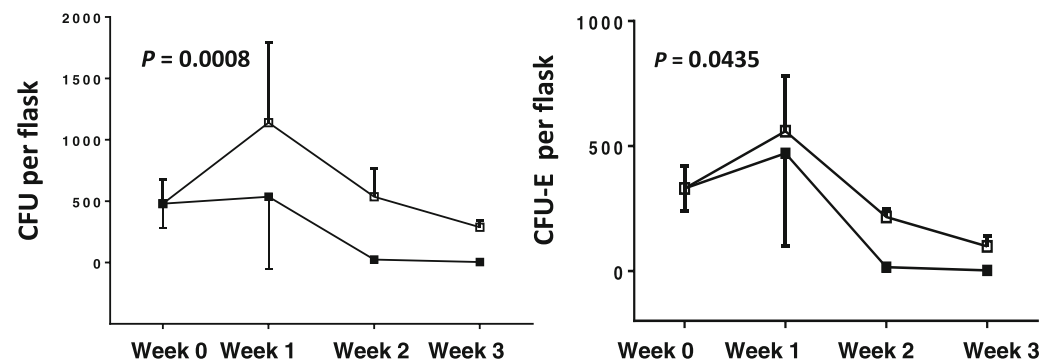

b Recharges with UCB-derived CD34+ cells
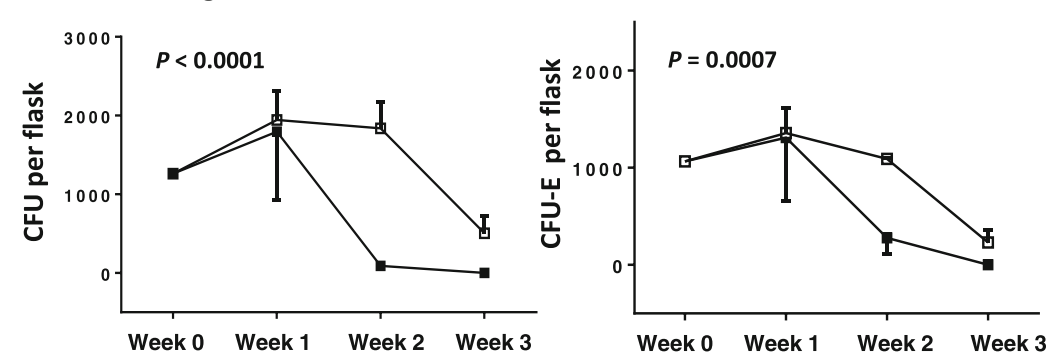

Feeder layers of WJ-MSCs

Feeder layers of BM-MSCs

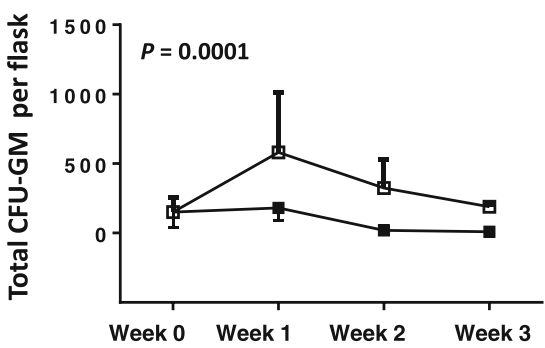

Feeder layers of WJ-MSCs

Feeder layers of BM-MSCs

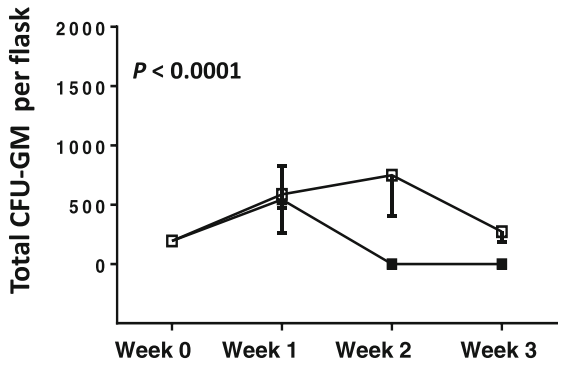

Fig. 7 The hematopoiesis-supporting capacity of WJ-MSCs and BM-MSCs. Confluent stromal layers from WJ-MSCs $(n=6)$ and BM-MSCs $(n=6)$ at P2 grown in $25 \mathrm{~cm}^{2}$ flasks, were irradiated and recharged with normal allogeneic BM-derived (a) or UCB-derived (b) CD34 cells. At weekly intervals for a total of 3 weeks, cultures were fed by demi-depopulation and the non-adherent cells were assayed for clonogenic progenitor cells in methylcellulose culture medium. The graphs depict the number (mean \pm 1 standard deviation) of total colony-forming units (CFU), erythroid colony-forming units (CFU-E) and total granulocyte-macrophage colony-forming units (CFU-GM) time course obtained from the non-adherent cell fraction of recharged WJ-MSC or BM-MSC cultures. Comparison of mean colony numbers over the 3-week culture between WJ- and BM-MSC feeder layers was performed by the two-way analysis of variance and the $P$ values are depicted. Abbreviations: BM-MSCS bone marrow-mesenchymal stem cells, UCB umbilical cord blood, WJ-MSCs Wharton's jelly-mesenchymal stem cells

Ex vivo expanded WJ-MSCs showed similar fibroblastlike morphology and immunophenotypic characteristics compared to BM-MSCs. However, the growth rate of WJ-MSCs over passages was significantly increased compared to BM-MSCs, as was demonstrated by the PD time through passages and the metabolic activity of cells at a representative passage (P2). Our data on the survival characteristics of MSCs suggest that the differential growth of the two MSC populations cannot be attributed to differences in the rate of apoptotic cell death but may be associated to the increased proliferation of WJ-MSCs, at least in part. Indeed, significant higher proportion of WJ-MSCs was found in S and G2/M phase of cell cycle in flow cytometric analysis, compared to BM-MSCs, and this finding was associated with alterations in the expression of genes involved in G1/S and G2/M transition.

Table 2 Cytokines in MSC culture supernatants

\begin{tabular}{|c|c|c|c|c|c|c|c|}
\hline \multirow[t]{2}{*}{ Cytokines $^{a}$} & \multicolumn{3}{|c|}{$\begin{array}{l}\text { WJ-MSC culture supernatants } \\
(\mathrm{n}=18)\end{array}$} & \multicolumn{4}{|c|}{$\begin{array}{l}\text { BM-MSC culture supernatants } \\
(n=18)\end{array}$} \\
\hline & $\mathrm{P} 2$ & P6 & P10 & P2 & P6 & P10 & $P$ value \\
\hline FLT3L (ng/mL) & $13.79 \pm 0.75$ & $14.35 \pm 1.24$ & $13.74 \pm 0.87$ & $13.77 \pm 0.96$ & $14.70 \pm 62.33$ & $13.43 \pm 0.45$ & N.S. \\
\hline SDF1-a (ng/mL) & $1189.00 \pm 380.40$ & $1159.00 \pm 401.00$ & $1032.00 \pm 269.00$ & $2074.00 \pm 1247.00$ & $1607.00 \pm 698.00$ & $1369.00 \pm 420.60$ & 0.0012 \\
\hline $\mathrm{G}-\mathrm{CSF}(\mathrm{ng} / \mathrm{mL})$ & $108.30 \pm 100.70$ & $578.00 \pm 409.80$ & $388.00 \pm 360.00$ & N.D. & N.D. & N.D & $<0.0001$ \\
\hline GM-CSF (ng/mL) & $12.19 \pm 9.46$ & $145.20 \pm 135.60$ & $37.70 \pm 13.58$ & N.D. & N.D. & N.D. & 0.0002 \\
\hline
\end{tabular}

Cytokine measurements have been performed by means of an enzyme-linked immunosorbent assay (ELISA). Comparisons in cytokine levels between WJ-MSCs and BM-MSC supernatants through passages (P) P2-P6-P10, were performed by two-way analysis of variance and the statistically significant $P$ values are shown Abbreviations: WJ-MSCS Wharton's jelly-mesenchymal stem cells, BM-MSCs bone marrow-derived mesenchymal stem cells, $P$ passage, FLT3L FMS-like tyrosine kinase 3 ligand, SDF-1a stromal cell-derived factor-1a, G-CSF granulocyte colony-stimulating factor, GM-CSF granulocyte-macrophage colony -stimulating factor, N.S. no statistically significant difference, N.D. not detected

${ }^{a}$ The data are expressed as means \pm 1 standard deviation 
Specifically, an upregulation of CCND2, CDC25A, CCNA2, CCNB1, CDC28, CKS2, CDC25C, CDC20 and $A U R K B$ was found in WJ-MSCs compared to BM-MSCs that was associated with downregulation of the antiapoptotic gene $B C L 2$, which mediates delayed transition into $\mathrm{S}$ phase, and downregulation of $C D K N 1 B$, which is implicated in G1 arrest. To the best of our knowledge, comparative analysis of cell cycle-related genes between WJ-MSCs and BM-MSCs has not been previously reported and the results give an explanation for the increased growth rate of the WJ-MSCs in comparison to their BM counterparts.

Since replicative senescence has been associated with defective cellular proliferative capacity, we investigated whether the inferior growth of BM-MSCs throughout culture might be due to increased propensity to cell senescence compared to WJ-MSCs. However, the expression of three cell cycle inhibitors which are usually upregulated in senescent cells, namely RB1, CDKN1A, $C D K N 2 A$, as well as the expression of the senescence associated-gene PARG1 known to increase during passages $[29,30]$, did not differ significantly between the two MSC populations at P2-P6-P10. TP53 was found overexpressed in WJ-MSCs compared to BM-MSCs. However, TP53 is not solely associated to cell senescence but also functions as a guard of the genome by inhibiting the cell cycle and therefore allowing the time for repair of potential DNA damage [31]. Based on the increased proliferation potential of WJ-MSCs, one may hypothesize that the upregulation of TP53 may act a safety mechanism, ensuring that these cells will not accumulate genomic instability during successive divisions.

Telomere length has been recognized as the main indicator of MSC replicative senescence [21, 32]. The mean telomere length did not differ significantly between WJand BM-MSCs through passages, suggesting further that cell senescence is unlikely to account for the inferior proliferative potential of BM-MSCs. In contrast to the aforementioned observations, the number of SA- $\beta$-galpositive cells was significantly higher in BM-MSC cultures compared to WJ-MSCs throughout passages. Nevertheless, SA- $\beta$-gal activity, although associated, is not completely specific for senescence [33,34]. Instead, it can give false-positive results when used as the sole method to identify senescence and should therefore be interpreted in combination with other biomarkers [33, 34]. However, given that the expression of SA- $\beta$-gal has been mostly associated with a non-proliferative state [34], the increased proportion of SA- $\beta$ gal-positive cells, may have a role in the decreased proliferative characteristics of BMcompared to WJ-MSCs.

The use of MSCs in cell-based therapies may require large-scale ex vivo expansion. To investigate whether long-term culture may influence the genetic stability of
WJ-MSCs, we performed cytogenetic analysis of WJand BM-MSCs through passages. Clonal cytogenetic aberrations were detected in WJ-MSCs from 2/18 (11.11\%) samples [del(1)(p34) and add(2)(p25.3)] and in BMMSCs from 3/18 (16.67\%) samples (trisomy 5). Notably in all cases, MSCs showed progressive growth arrest and eventually entered senescence. Our findings are in line with previous reports suggesting that BM-MSCs may occasionally develop genetic aberrations after long-term culture, without evidence of malignant transformation whatsoever [35-37]. As regards WJ-MSCs, contradictory results have been reported so far for the genomic stability of UC-derived MSCs [38-42]. The inconsistencies are probably related to the variability of MSC populations, culture conditions and methodologies (classical karyotype, FISH or comparative genomic hybridization array). Nevertheless, on the basis of our findings, BMMSCs and WJ-MSCs develop chromosomal aberrations at the same rate when expanded under the same experimental conditions and karyotypic analysis of cultureexpanded MSCs is reasonable before any application of cells in the clinic.

With regards to the ex vivo differentiation potential, WJ-MSCs displayed inferior adipogenic capacity compared to BM-MSCs, as evidenced by the Oil Red O staining and the expression of adipogenesis-specific genes PPARG and CEBPA. The WJ-MSCs also displayed reduced osteogenic potential compared to BM-MSCs, as evidenced by the lower expression of the osteogenesisrelated genes RUNX2, DLX5, ALP and OSC despite the similar Von Kossa and Alizarin Red staining. The above findings are in line with previous reports indicating reduced differentiation capacity of WJ-MSCs toward adipocytes and osteocytes [43-45]. In our study, we also showed decreased expression of osteogenesis- and adipogenesis-related genes in undifferentiated WJ-MSCs at P2 compared to BM-MSCs, suggesting an inferior lineage priming of WJ-MSCs to osteoblastic and adipocytic lineages.

Because mechanisms accounting for the weak differentiation potential of WJ-MSCs toward adipocytes and osteocytes have not been elucidated thus far and based on the established role of the WNT signaling pathway in MSC differentiation [46-49], we comparatively investigated the expression profile of genes associated with this pathway in BM- and WJ-MSCs. Twenty-five out of 84 genes were found to be differentially expressed between the two cell populations. Although the functional significance of these differences remains to be fully elucidated, we speculate that the differential expression of the WNT pathway-associated molecules has probably a role in the inferior osteogenic and adipogenic potential of WJMSCs compared to BM-MSCs. Indeed, WNT3A, $-5 A$ and $-7 B$ known to promote osteogenesis $[46,47]$ were 
downregulated, whereas the canonical WNT pathway negative regulator $D K K 1$, known to inhibit osteogenesis [46], was upregulated in WJ-MSCs. Furthermore, based on the finding of significant downregulation of WISP1 in WJ- compared to BM-MSCs and also on previously reported data on the role of this molecule in osteoblastic differentiation in vitro and osteogenesis in vivo [26, 27], we studied its significance in the osteogenic differentiation of WJ-MSCs. The upregulation of WISP1 expression during the osteogenic differentiation of WJ-MSCs in association with the finding that treatment with rhWISP1 resulted in induction of the osteogenic potential of WJ-MSCs, as was evidenced by the upregulation of RUNX2, ALP and OSC expression, corroborates the assumption that the downregulation of WISP1 in WJMSCs compared to BM-MSCs is probably implicated in their inferior differentiation capacity toward the osteoblastic lineage.

The implication of WNT signaling pathway in the adipogenic differentiation of MSCs has been previously reported $[48,49]$. In this context, and because $s F R P 4$, a molecule known to be implicated in adipogenesis $[25,50]$, was found significantly downregulated in WJ-MSCs compared to BM-MSCs, we investigated the potential role of $s F R P 4$ in the adipogenic differentiation of WJ-MSCs. The increase of $s F R P 4$ mRNA during the adipogenic differentiation of WJ-MSCs in association with the finding that treatment with rh-sFRP4 resulted in induction of the adipogenic potential of WJ-MSCs, as was indicated by the upregulation of PPARG and CEBPA expression, favors the hypothesis that the downregulation of SFRP4 in WJ-MSCs compared to BM-MSCs is probably implicated in their inferior differentiation capacity toward adipocytes. The involvement of WISP1 and SFRP4 on the differentiation potential of WJ-MSCs provide novel insights on the role of WNT signaling in this MSC population.

Limited data are currently available on the hematopoiesis-supporting capacity of WJ-MSCs compared to BM-MSCs $[51,52]$. To probe this issue, we recharged in parallel WJ- and BM-MSC stromal layers with the same batch of UCB- or BM-derived CD34 ${ }^{+}$cells and subsequently evaluated the capacity of the stromal cells to support the clonogenic potential of the supernatant $\mathrm{CD}_{3} 4^{+}$cells, over 3 weeks. We found that feeder layers derived from WJ-MSCs displayed an inferior potential to support myeloid and erythroid colony formation by the supernatant hematopoietic cells, compared to BMMSCs. This observation corroborates a previous report [51], whereas it contradicts another study which detected no difference in the hematopoiesis-supporting function of UC- and BM-MSCs [52]. This controversy might be attributed, at least in part, to the fact that in the latter study MSCs from the whole UC were used as feeder layers.
The observed differences in the hematopoiesissupporting capacity of WJ- compared to BM-MSCs might be due, at least in part, to the different cytokine production. In line with a previous report [53], we have also shown that WJ-MSCs secrete lower SDF- $1 \alpha$ and higher G-CSF and GM-CSF amounts, compared to BM-MSCs. We assume that the increased levels of G-CSF and GM-CSF may not be able to functionally counterbalance the decreased production of SDF- $1 \alpha$ by the WJMSCs [54, 55]. The differential expression of the WNT pathway-related molecules between WJ- and BM-MSCs may also have a role in the different hematopoiesissupporting function of these two MSC populations. For example, the canonical WNT pathway, which was downregulated in WJ-MSCs, has been reported to display a positive effect [56], whereas the canonical pathway inhibitor DKK1, which was upregulated in WJ-MSCs, has been reported to exert a negative effect on the hematopoietic progenitor cell growth [57]. Interestingly, both WJ- and BM-MSCs were less effective in supporting the growth of the BM-derived than the UCB-derived $\mathrm{CD} 34^{+}$cells probably because the $\mathrm{BM}$ hematopoietic stem/progenitor cell fraction contains less primitive and fewer clonogenic cells compared to UCB [58].

\section{Conclusions}

Overall, we have performed a rigorous, head-to-head comparison of ex vivo expanded WJ-MSCs and BMMSCs and have provided insights in their immunophenotypic, survival, proliferative and cytogenetic characteristics, their differentiation potential and their capacity to support hematopoiesis. We have provided, for the first time, evidence for differential expression of cell cycle and WNT pathway-related molecules that may significantly contribute to the observed differences in the biological properties of the two MSC populations. We have also pointed out the prominent role of the WNT pathwayrelated molecules WISP1 and SFRP4 in the osteogenic and adipogenic differentiation potential of WJ-MSCs. These data contribute to the better characterization of WJ-MSCs and BM-MSCs in view of their use in the clinic and even encourage the probability of engineering the WNT pathway in WJ-MSCs for specific clinical applications.

\footnotetext{
Abbreviations

7-AAD: 7-aminoactinomycin D; ALP: Alkaline phosphatase; BM: Bone marrow; CDKN: Cyclin-dependent kinase; CEBPA: CCAAT/enhancerbinding protein alpha; CFU: Colony-forming units; DLX5: Distal-less homeobox protein 5; FC: Fold change; FLT3L: FMS-like tyrosine kinase 3 ligand; GAPDH: Glyceraldehyde-3-phosphate dehydrogenase; G-CSF: Granulocyte colony-stimulating factor; GM-CSF: Granulocytemacrophage colony-stimulating factor; MSC: Mesenchymal stem cells; MTT: 3-[4,5-dimethylthiazol-2-yl]-2,5 diphenyl tetrazolium bromide; NACs: Non-adherent cells; OSC: Osteocalcin; P: Passage; PARG1: Phosphate-associated RhoGAP protein-tyrosine; PD: Population doubling; PPARG: Peroxisome proliferator activated receptor- $\gamma_{;}$RB1: Retinoblastoma; RT-PCR: Real-time reverse transcription polymerase chain reaction; RUNX2: Runt-related
} 
transcription factor 2; SA- $\beta$-gal: Senescence-associated $\beta$-galactosidase; SDF-1a:

Stromal cell-derived factor-1a; sFRP4: Secreted frizzled related protein 4; UC: Umbilical cord; UCB: Umbilical cord blood; WISP1: WNT1-induciblesignaling pathway protein 1; WJ: Wharton's jelly

\section{Acknowledgements}

We are grateful to the patients and mothers who gave their consent for donation of BM and UC samples, respectively.

\section{Funding}

This work was supported by the European Commission FP7 programme "Translational Potential" (TransPOT; EC contract number 285948) and a grant by the General Secretariat of Research and Technology, Greece (UMBISTEM; SYNERGASIA 11SYN_10_668).

\section{Availability of data and materials}

The datasets supporting the results of this article are included within the article.

\section{Authors' contributions}

AKB performed the experiments, analyzed and interpreted the data and drafted the manuscript. CP supervised and interpreted the experiments, analyzed the data and contributed in the drafting of the manuscript. DK provided the umbilical cord samples and critically reviewed the manuscript. KIP, AD and EK performed experiments. IM performed experiments and critically reviewed the manuscript. KA and GK provided the bone marrow samples and critically reviewed the manuscript. HAP designed the study and wrote the manuscript. All authors read and approved the final manuscript.

\section{Competing interests}

The authors declare that they have no competing interests.

\section{Consent for publication}

Not applicable.

\section{Ethics approval and consent to participate}

The study has been approved by the Ethics Committee of the University Hospital of Heraklion (reference number 1724/20-03-2012) and informed consent for the UC and the BM donation was obtained from the neonate mothers and the individuals undergoing surgery, respectively, according to the Helsinki Protocol.

\section{Publisher's Note}

Springer Nature remains neutral with regard to jurisdictional claims in published maps and institutional affiliations.

\section{Author details}

'University of Crete School of Medicine, Heraklion, Greece. ${ }^{2}$ Graduate Program "Molecular Basis of Human Disease", University of Crete School of Medicine, Heraklion, Greece. ${ }^{3}$ Department of Obstetrics and Gynecology, University of Crete School of Medicine, Heraklion, Greece. ${ }^{4}$ Department of Orthopedics and Traumatology, University of Crete School of Medicine, Heraklion, Greece.

Received: 1 February 2017 Revised: 14 March 2017

\section{Accepted: 5 April 2017 Published online: 26 April 2017}

\section{References}

1. Sensebe L, Krampera M, Schrezenmeier H, Bourin P, Giordano R. Mesenchymal stem cells for clinical application. Vox Sang. 2010;98:93-107.

2. Batsali AK, Kastrinaki MC, Papadaki HA, Pontikoglou C. Mesenchymal stem cells derived from Wharton's ielly of the umbilical cord: biological properties and emerging clinical applications. Curr Stem Cell Res Ther. 2013;8:144-55.

3. Wegmeyer H, Broske AM, Leddin M, Kuentzer K, Nisslbeck AK, Hupfeld J, et al. Mesenchymal stromal cell characteristics vary depending on their origin. Stem Cells Dev. 2013;22:2606-18.

4. Troyer $\mathrm{DL}$, Weiss ML. Wharton's jelly-derived cells are a primitive stromal cell population. Stem Cells. 2008;26:591-99.

5. Subramanian A, Fong CY, Biswas A, Bongso A. Comparative characterization of cells from the various compartments of the human umbilical cord shows that the Wharton's jelly compartment provides the best source of clinically utilizable mesenchymal stem cells. PloS One. 2015;10, e0127992.
6. Kastrinaki MC, Sidiropoulos P, Roche S, Ringe J, Lehman S, Kritikos H, et al. Functional, molecular and proteomic characterisation of bone marrow mesenchymal stem cells in rheumatoid arthritis. Ann Rheum Dis. 2008;67:741-49.

7. Pavlaki K, Pontikoglou CG, Demetriadou A, Batsali AK, Damianaki A, Simantirakis $E$, et al. Impaired proliferative potential of bone marrow mesenchymal stromal cells in patients with myelodysplastic syndromes is associated with abnormal WNT signaling pathway. Stem Cells Dev. 2014;23: 1568-81.

8. Chatzinikolaidou M, Kaliva M, Batsali A, Pontikoglou C, Vamvakaki M. Wharton's jelly mesenchymal stem cell response on chitosan-graft-poly (epsilon-caprolactone) copolymer for myocardium tissue engineering. Curr Pharm Des. 2014;20:2030-39.

9. Philpott NJ, Turner AJ, Scopes J, Westby M, Marsh JC, Gordon-Smith EC. The use of 7-amino actinomycin D in identifying apoptosis: simplicity of use and broad spectrum of application compared with other techniques. Blood. 1996;87:2244-51.

10. Pontikoglou C, Kastrinaki MC, Klaus M, Kalpadakis C, Katonis P, Alpantaki K, et al, Study of the quantitative, functional, cytogenetic, and immunoregulatory properties of bone marrow mesenchymal stem cells in patients with B-cell chronic lymphocytic leukemia. Stem Cells Dev. 2013;22:1329-41.

11. Cristofalo VJ, Allen RG, Pignolo RJ, Martin BG, Beck JC. Relationship between donor age and the replicative lifespan of human cells in culture: a reevaluation. Proc Natl Acad Sci U S A. 1998:95:10614-19.

12. Pavlaki Kl, Kastrinaki MC, Klontzas M, Velegraki M, Mavroudi I, Papadaki HA. Abnormal telomere shortening of peripheral blood mononuclear cells and granulocytes in patients with chronic idiopathic neutropenia. Haematologica. 2012;97:743-50.

13. Debacq-Chainiaux F, Boilan E, Le Dedessus MJ, Weemaels G, Toussaint O. p38(MAPK) in the senescence of human and murine fibroblasts. Adv Exp Med Biol. 2010;694:126-37.

14. Delorme B, Charbord P. Culture and characterization of human bone marrow mesenchymal stem cells. Methods Mol Med. 2007;140:67-81.

15. Klaus M, Stavroulaki E, Kastrinaki MC, Fragioudaki P, Giannkiou K, Psyllaki M, et al. Reserves, functional, immunoregulatory, and cytogenetic properties of bone marrow mesenchymal stem cells in patients with myelodysplastic syndromes. Stem Cells Dev. 2010;19:1043-54.

16. Shaffer LG, McGowan-Jordan J, Schmid M, International Standing Committee on Human Cytogenetic Nomenclature. ISCN 2013: an international system for human cytogenetic nomenclature. Basel: Karger; 2013.

17. Kouvidi E, Stratigi A, Batsali A, Mavroudi I, Mastrodemou S, Ximeri M, et al. Cytogenetic evaluation of mesenchymal stem/stromal cells from patients with myelodysplastic syndromes at different time-points during ex vivo expansion. Leuk Res. 2016:43:24-32.

18. Coutinho LH, Gilleece MH, De Wynter EA, Will A, Testa NG. Clonal and longterm cultures using human bone marrow. In: Testa NG, Molineux G, editors. Haemopoiesis: a practical approach. Oxford: Oxford University Press; 1993. p. 75-106.

19. Papadaki HA, Eliopoulos AG, Kosteas T, Gemetzi C, Damiamaki A, Koutala H, et al. Impaired granulocytopoiesis in patients with chronic idiopathic neutropenia is associated with increased apoptosis of bone marrow myeloid progenitor cells. Blood. 2003;101:2591-600.

20. Lee BY, Han JA, Im JS, Morrone A, Johung K, Goodwin EC, et al. Senescence-associated beta-galactosidase is lysosomal beta-galactosidase. Aging Cell. 2006;5:187-95.

21. Wagner W, Bork S, Lepperdinger G, Joussen S, Ma N, Strunk D, et al. How to track cellular aging of mesenchymal stromal cells? Aging (Albany NY). 2010:2:224-30.

22. Ling $L$, Nurcombe $V$, Cool SM. Wnt signaling controls the fate of mesenchymal stem cells. Gene. 2009:433:1-7.

23. Lien WH, Fuchs E. Wnt some lose some: transcriptional governance of stem cells by Wnt/beta-catenin signaling. Genes Dev. 2014;28:1517-32.

24. Kawano $Y$, Kypta R. Secreted antagonists of the Wnt signalling pathway. J Cell Sci. 2003;116:2627-34.

25. Visweswaran M, Schiefer L, Arfuso F, Dilley RJ, Newsholme P, Dharmarajan A Wnt antagonist secreted frizzled-related protein 4 upregulates adipogenic differentiation in human adipose tissue-derived mesenchymal stem cells. Plos One. 2015;10, e0118005.

26. Inkson CA, Ono M, Kuznetsov SA, Fisher LW, Robey PG, Young MF. TGFbeta1 and WISP-1/CCN-4 can regulate each other's activity to cooperatively control osteoblast function. J Cell Biochem. 2008;104:1865-78. 
27. Ono M, Inkson CA, Kilts TM, Young MF. WISP-1/CCN4 regulates osteogenesis by enhancing BMP-2 activity. J Bone Miner Res. 2011;26:193-208.

28. Inkson CA, Ono M, Bi Y, Kuznetsov SA, Fisher LW, Young MF. The potential functional interaction of biglycan and WISP-1 in controlling differentiation and proliferation of osteogenic cells. Cells Tissues Organs. 2009;189:153-7.

29. Kuilman T, Michaloglou C, Mooi WJ, Peeper DS. The essence of senescence. Genes Dev. 2010;24:2463-79.

30. Schallmoser K, Bartmann C, Rohde E, Bork S, Guelly C, Obenauf AC, et al. Replicative senescence-associated gene expression changes in mesenchymal stromal cells are similar under different culture conditions. Haematologica. 2010;95:867-74.

31. Zhao T, Xu Y. p53 and stem cells: new developments and new concerns. Trends Cell Biol. 2010;20:170-5.

32. Bernadotte A, Mikhelson VM, Spivak IM. Markers of cellular senescence. Telomere shortening as a marker of cellular senescence. Aging (Albany NY). 2016;8:3-11.

33. Debacq-Chainiaux F, Erusalimsky JD, Campisi J, Toussaint O. Protocols to detect senescence-associated beta-galactosidase (SA-betagal) activity, a biomarker of senescent cells in culture and in vivo. Nat Protoc. 2009;4:1798-806.

34. Matjusaitis M, Chin G, Sarnoski EA, Stolzing A. Biomarkers to identify and isolate senescent cells. Ageing Res Rev. 2016;29:1-12

35. Tarte K, Gaillard J, Lataillade JJ, Fouillard L, Becker M, Mossafa H, et al. Clinical-grade production of human mesenchymal stromal cells: occurrence of aneuploidy without transformation. Blood. 2010;115:1549-53.

36. Capelli C, Pedrini O, Cassina G, Spinelli O, Salmoiraghi S, Golay J, et al. Frequent occurrence of non-malignant genetic alterations in clinical grade mesenchymal stromal cells expanded for cell therapy protocols. Haematologica. 2014;99(6):e94-7.

37. Borgonovo T, Solarewicz MM, Vaz IM, Daga D, Rabelatto CL, Senegaglia AC, et al. Emergence of clonal chromosomal alterations during the mesenchymal stromal cell cultivation. Mol Cytogenet. 2015;8:94

38. Borghesi A, Avanzini MA, Novara F, Mantelli M, Lenta F, Achille V, et al. Genomic alterations in human umbilical cord-derived mesenchymal stromal cells call for stringent quality control before any possible therapeutic approach. Cytotherapy. 2013;15:1362-73.

39. Wang Y, Zhang Z, Chi Y, Zhang Q, Xu F, Yang Z, et al. Long-term cultured mesenchymal stem cells frequently develop genomic mutations but do not undergo malignant transformation. Cell Death Dis. 2013;4, e950.

40. Nekanti U, Rao VB, Bahirvani AG, Jan M, Totey S, Ta M. Long-term expansion and pluripotent marker array analysis of Wharton's jelly-derived mesenchymal stem cells. Stem Cells Dev. 2010;19:117-30.

41. Capelli C, Gotti E, Morigi M, Rota C, Weng L, Dazzi F, et al. Minimally manipulated whole human umbilical cord is a rich source of clinical-grade human mesenchymal stromal cells expanded in human platelet lysate. Cytotherapy. 2011;13:786-801.

42. Chen G, Yue A, Ruan Z, Yin Y, Wang R, Ren Y, et al. Human umbilical cordderived mesenchymal stem cells do not undergo malignant transformation during long-term culturing in serum-free medium. PloS One. 2014;9, e98565.

43. Karahuseyinoglu S, Cinar O, Kilic E, Kara F, Akay CG, Demiralp DO, et al. Biology of stem cells in human umbilical cord stroma: in situ and in vitro surveys. Stem Cells. 2007;25:319-31.

44. Hsieh JY, Fu YS, Chang SJ, Tsuang YH, Wang HW. Functional module analysis reveals differential osteogenic and stemness potentials in human mesenchymal stem cells from bone marrow and Wharton's jelly of umbilical cord. Stem Cells Dev. 2010;19:1895-910.

45. Cavallo C, Cuomo C, Fantini S, Ricci F, Tazzari PL, Lucarelli E, et al. Comparison of alternative mesenchymal stem cell sources for cell banking and musculoskeletal advanced therapies. J Cell Biochem. 2011;112:1418-30.

46. Pinzone JJ, Hall BM, Thudi NK, Vonau M, Qiang YW, Rosol TJ, et al. The role of Dickkopf-1 in bone development, homeostasis, and disease. Blood. 2009; 113:517-25

47. Visweswaran M, Pohl S, Arfuso F, Newsholme P, Dilley R, Pervaiz S, et al. Multi-lineage differentiation of mesenchymal stem cells - To Wnt, or not Wnt. Int J Biochem Cell Biol. 2015;68:139-47.

48. Christodoulides C, Lagathu C, Sethi JK, Vidal-Puig A. Adipogenesis and WNT signalling. Trends Endocrinol Metabol. 2009;20:16-24

49. Laudes M. Role of WNT signalling in the determination of human mesenchymal stem cells into preadipocytes. J Mol Endocrinol. 2011;46:65-72.

50. Park JR, Jung JW, Lee YS, Kang KS. The roles of Wnt antagonists Dkk1 and sFRP4 during adipogenesis of human adipose tissue-derived mesenchymal stem cells. Cell Prolif. 2008;41:859-74.
51. Bakshi T, Zabriskie RC, Bodie S, Kidd S, Ramin S, Paganessi LA, et al. Mesenchymal stem cells from Wharton's jelly of umbilical cord segments provide stromal support for the maintenance of cord blood hematopoietic stem cells during long-term ex vivo culture. Transfusion. 2008;48:2638-44.

52. Lu LL, Liu YJ, Yang SG, Zhao QJ, Wang X, Gong W, et al. Isolation and characterization of human umbilical cord mesenchymal stem cells with hematopoiesis-supportive function and other potentials. Haematologica. 2006;91:1017-26.

53. Friedman R, Betancur M, Boissel L, Tuncer H, Cetrulo C, Klingemann H. Umbilical cord mesenchymal stem cells: adjuvants for human cell transplantation. Biol Blood Marrow Transplant. 2007:13:1477-86.

54. Olofsson TB. Growth regulation of hematopoietic cells. An overview. Acta Oncol. 1991;30:889-902.

55. Rosu-Myles M, Bhatia M. SDF-1 enhances the expansion and maintenance of highly purified human hematopoietic progenitors. Hematol J. 2003:4:137-45.

56. Cain CJ, Manilay JO. Hematopoietic stem cell fate decisions are regulated by Wnt antagonists: comparisons and current controversies. Exp Hematol. 2013;41:3-16

57. Fleming HE, Janzen V, Lo CC, Guo J, Leahy KM, Kronenberg HM, et al. Wnt signaling in the niche enforces hematopoietic stem cell quiescence and is necessary to preserve self-renewal in vivo. Cell Stem Cell. 2008;2:274-83.

58. Hordyjewska A, Popiolek L, Horecka A. Characteristics of hematopoietic stem cells of umbilical cord blood. Cytotechnology. 2015;67:387-96.

\section{Submit your next manuscript to BioMed Central and we will help you at every step:}

- We accept pre-submission inquiries

- Our selector tool helps you to find the most relevant journal

- We provide round the clock customer support

- Convenient online submission

- Thorough peer review

- Inclusion in PubMed and all major indexing services

- Maximum visibility for your research

Submit your manuscript at www.biomedcentral.com/submit
Biomed Central 